\title{
Photoionization of a strongly polarizable target
}

\author{
A V Korol ${ }^{1,2}$ and A V Solov'yov ${ }^{1}$ \\ ${ }^{1}$ Frankfurt Institute for Advanced Studies, Johann Wolfgang Goethe-Universität, \\ Ruth-Moufang-Str. 1, 60438 Frankfurt am Main, Germany \\ 2 Department of Physics, St.Petersburg State Maritime Technical University, \\ Leninskii prospect 101, St. Petersburg 198262, Russia \\ E-mail: korol@fias.uni-frankfurt.de, solovyov@fias.uni-frankfurt.de
}

\begin{abstract}
We demonstrate that the angular distribution of photoelectrons from a strongly polarizable target exposed to a laser field can deviate noticeably from the prediction of conventional theory. Even within the dipole-photon approximation the profile of distribution is modified due to the action of the field of alternating dipole moment induced at the residue by the laser field. This effect, being quite sensitive to the dynamic polarizability of the residue and to its geometry, depends also on the intensity and frequency of the laser field. Numerical results, presented for sodium cluster anions, demonstrate that dramatic changes to the profile occur for the photon energies in vicinities of the plasmon resonances, where the effect is enhanced due to the increase in the residue polarizability. Strong modifications of the characteristics of a singlephoton ionization process can be achieved by applying laser fields of comparatively low intensities $I_{0} \sim 10^{10}-10^{11} \mathrm{~W} / \mathrm{cm}^{2}$.
\end{abstract}

\section{Introduction}

In this paper we demonstrate that the profile of angular distribution of electrons emitted in the process of a single-photon ionization from a strongly polarizable target (in particular, a metal cluster anion) exposed to a laser field can be noticeably modified due to the action of the field $\mathcal{U}(\mathbf{r}, t)$ of alternating dipole moment $\mathbf{D}(t)$ induced at the residue by the laser field. The field $\mathcal{U}(\mathbf{r}, t)$ acts on the escaping electron and, being dependent on the polarization vector of the laser field, brings additional dependence of the cross section on the emission angle. The degree of this dependence is determined by the magnitude of $\mathbf{D}(t)$ which, in turn, is proportional to the residue's dynamic polarizability. Therefore, for a target with large polarizability the effect can be very pronounced even for laser fields of low intensities.

The effect of the laser-field-induced dipole moment in atomic photoionization process was discussed in [1] (where one finds also the references to the earlier works by the authors) and, more recently, in [2]. Both papers were devoted to the study of multiphoton detachment of electrons from atomic negative ions. In [1] the process was analyzed by means of the perturbation theory. Numerical analysis was carried out for halogen and alkali metal anions, and it demonstrated, that the induced dipole moment 
influences the magnitude of total cross section of the many-photon detachment. The paper [2] dealt with the process of a strong-field many-photon detachment of an atomic anion. The non-perturbative adiabatic theory, developed in [3], was modified to account for the field of the induced dipole moment. The numerical calculations, performed for $\mathrm{Rb}^{-}$and $\mathrm{Cs}^{-}$, showed the increase of the detachment rate and essential modification of the angular distribution.

In our paper we investigate the influence of the induced dipole moment on the angular and spectral distributions of photoelectrons in the process of single-photon ionization (or detachment) occurring in the weak-field regime. Numerical results are presented for sodium cluster anions $\mathrm{Na}_{N}^{-}$with $N=10^{1} \ldots 10^{2}$. The corresponding residue clusters are characterized by large values of the dynamic dipole polarizability $\alpha(\omega)$, which in the vicinity of plasmon resonances, i.e. at $\omega=2 \ldots 3 \mathrm{eV}$, can be as large as $10^{4} \ldots 10^{5}$ a.u., which by far exceed the maximum values of $\alpha(\omega)$ of individual atoms (e.g., $\alpha(\omega) \approx 400$ a.u. for Cs at the photon energy $0.47 \mathrm{eV}[2])$.

We demonstrate that dramatic changes to the profile of angular distribution occur in vicinities of the plasmon resonances and for comparatively low intensities $\sim 10^{10}-10^{11}$ $\mathrm{W} / \mathrm{cm}^{2}$ of the laser field. These are exactly the ranges used in recent experimental studies $[4,5]$ of the photodetachment from various metal cluster anions carried out by means of angle resolved photoelectron spectroscopy. Therefore, the experimental test of the predicted effects seems to be feasible.

The atomic system of units, $e=m=\hbar=1$ is used throughout the paper.

\section{Theoretical framework}

\subsection{Setting the problem}

Consider the process of photoionization of a strongly polarizable target (a cluster, a fullerene, a complex molecule, etc) by spatially homogenous linearly polarized laser field

$$
\mathbf{E}(t)=\mathbf{E}_{0} \cos \omega t=\frac{1}{2}\left(\mathbf{E}_{0} \mathrm{e}^{-\mathrm{i} \omega t}+\mathbf{E}_{0} \mathrm{e}^{\mathrm{i} \omega t}\right)
$$

We assume, that the field wavelength $\lambda$ greatly exceeds the characteristic size $R$ of the target, $\lambda \gg R$. Thus, the process can be treated within the dipole-photon limit.

The laser field ionizes the target and polarizes the residue. Therefore, in addition to other fields (the static potential of the residue, the long-range polarizational potential) the escaping photoelectron feels the field due to the oscillating dipole moment $\mathbf{D}(t)$ induced at the residue:

$$
\mathcal{U}(\mathbf{r}, t)=-\frac{\mathbf{D}(t) \mathbf{r}}{r^{3}} .
$$

To simplify the consideration we assume, that the residue is spherically symmetric, so that its tensor of polarizability contains only a scalar part which is notated as $\alpha(\omega)$. Then, the moment $\mathbf{D}(t)$ reads

$$
\mathbf{D}(t)=\mathbf{d} \mathrm{e}^{-\mathrm{i} \omega t}+\mathbf{d}^{*} \mathrm{e}^{\mathrm{i} \omega t},
$$


where $\mathbf{d} \equiv \mathbf{d}(\omega)=\alpha(\omega) \mathbf{E}_{0} / 2$. The field of the induced dipole can be written as follows:

$$
\mathcal{U}(\mathbf{r}, t)=U(\mathbf{r}) \mathrm{e}^{-\mathrm{i} \omega t}+U^{*}(\mathbf{r}) \mathrm{e}^{\mathrm{i} \omega t},
$$

with $U(\mathbf{r})=\mathbf{d r} / r^{3}$. This field is dependent on the direction of $\mathbf{E}_{0}$, therefore, the angular distribution of photoelectrons acquires additional dependence on the polarization of the laser field. The degree of this dependence is determined by the magnitude of $\mathbf{D}(t)$, which is proportional to the residue dynamic dipole polarizability. Hence, for a target with large polarizability the modification of the angular distribution due to the induced dipole moment can become noticeable even for a laser field of a comparatively low intensity.

\subsection{The perturbation series}

Let us clearly state the conditions which we impose on the parameters of the laser field. These include the following inequalities:

$$
\text { (I) } E_{0} \ll E_{\text {int }}, \quad \text { (II) } \varepsilon \gg \frac{E_{0}^{2}}{4 \omega^{2}}, \quad \text { (III) } \omega>\left|\varepsilon_{\mathrm{i}}\right| .
$$

The first condition implies that the laser field strength is much smaller than the strength $E_{\text {int }}$ of the internal field in the target. This condition allows one to ignore the laser field when constructing the wavefunction of the bound electron with the binding energy $\varepsilon_{\mathrm{i}}<0$. Numerical results presented in section 3 refer to the laser field strength $E_{0} \sim 10^{-3}$ a.u. $\sim 10^{6} \mathrm{~V} / \mathrm{cm}$.

The second condition states that the energy $\varepsilon$ of the escaping electron is large compared to the ponderomotive shift due to the wiggling in the laser field. Owing to this condition we do not "dress" the photoelectron wavefunction with the laser field.

The last inequality in (5) indicates that the photon energy exceeds the ionization threshold of the bound electron. Therefore, the process of a single-photon ionization is allowed for which the energy conservation law reads

$$
\varepsilon=\varepsilon_{\mathrm{i}}+\omega .
$$

Combining the conditions (I) and (III) one demonstrates that the following inequality is met: $\gamma \equiv \omega \kappa / E_{0} \gg 1$ where $\gamma$ is a so-called Keldysh parameter, and $\kappa=\left(2\left|\varepsilon_{\mathrm{i}}\right|\right)^{1 / 2}$. The limit $\gamma \gg 1$ defines a weak field regime (see, e.g. [3]). Thus, in this paper we investigate the influence of the induced dipole moment $\mathbf{D}(t)$ on the angular and spectral distributions of photoelectrons in the process of single-photon ionization (or detachment) occurring in the weak field regime. Earlier the role of $\mathbf{D}(t)$ was studied in the process of many-photon detachment (i.e., when $\omega \ll\left|\varepsilon_{\mathrm{i}}\right|$ ) from atomic negative ions. It was done in the weak-field [1] and in the strong-field [2] limits.

Let us note, that the weak field condition (II) does not immediately imply the applicability of the perturbative approach with respect to $\mathcal{U}(\mathbf{r}, t)$. Indeed, from (2)-(4) follows that $|\mathcal{U}(\mathbf{r}, t)| \sim E_{0}|\alpha(\omega)| / r^{2}$ exceeds $E_{0}$ by a factor $|\alpha(\omega)| / r^{2}$. For a strongly polarizable target this factor can be large enough in a wide range of radial distances $r>R$, and this might lead to a non-perturbative treatment of the action of $\mathcal{U}(\mathbf{r}, t)$. 
To carry out a quantitative analysis we first postulate that under certain conditions the field (4) can be considered as a time-dependent perturbation which modifies the wavefunction of the escaping electron. The criterion of applicability of this approach will be formulated in the course of calculations.

The matrix element $\mathcal{M}$, which describes a dipole-photon transition of the electron from the initial bound state $\psi_{i}(\mathbf{r}, t)=\psi_{i}(\mathbf{r}) \mathrm{e}^{-\mathrm{i} \varepsilon_{\mathrm{i}} t}$ to the final state $\psi_{f}(\mathbf{r}, t)=\psi_{\mathbf{p}}^{(-)}(\mathbf{r}) \mathrm{e}^{-\mathrm{i} \varepsilon t}$ with the asymptotic momentum $\mathbf{p}$ and energy $\varepsilon=p^{2} / 2$, can be written in the following form:

$$
\mathcal{M}=\mathcal{M}_{0}+\mathcal{M}_{1}+\mathcal{M}_{2}+\ldots
$$

The right-hand side of this equation represents the power series in $\mathcal{U}(\mathbf{r}, t)$. The structure of the terms of the series is illustrated by diagrams in figure 1 .

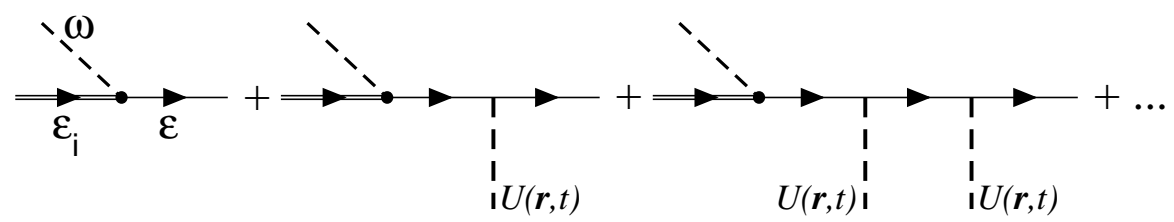

Figure 1. Diagrammatical representation of a single-photon transition amplitude in a form of perturbation series in $\mathcal{U}(\mathbf{r}, t)$ (indicated with the dashed vertical lines). The double line represents the initial bound state. The solid line stands for the escaping electron whose wavefunction is "dressed" with all static fields.

The zeroth order term $\mathcal{M}_{0}$ describes the direct process of photoionization standing for the matrix element of the operator $\mathbf{E}_{0} \mathbf{r} \exp (-\mathrm{i} \omega t) / 2$ :

$$
\mathcal{M}_{0}=\frac{1}{2} \int_{-\infty}^{\infty} \mathrm{d} t \psi_{f}^{*}(\mathbf{r}, t) \mathbf{E}_{0} \mathbf{r} \mathrm{e}^{-\mathrm{i} \omega t} \psi_{i}(\mathbf{r}, t)=2 \pi \delta\left(\varepsilon-\varepsilon_{\mathrm{i}}-\omega\right) M_{0},
$$

where

$$
M_{0}=\frac{1}{2} \int \mathrm{d} \mathbf{r} \psi_{\mathbf{p}}^{(-) *}(\mathbf{r})\left(\mathbf{E}_{0} \mathbf{r}\right) \psi_{i}(\mathbf{r}) .
$$

We assume that the wavefunction $\psi_{\mathbf{p}}^{(-)}(\mathbf{r})$ (whose asymptotic form is 'the plane wave + the incoming wave') corresponds to the state "dressed" with all static fields. The delta-function on the right-hand side of (8) expresses the energy conservation law (6).

The terms $\mathcal{M}_{n}$ with $n \geq 1$ are the corrections to the transition amplitude due to the $n$-times interaction of the photoelectron with $\mathcal{U}(\mathbf{r}, t)$. Since this field explicitly depends on $t$ (see (4)) then in each act of the interaction the electron energy is changed (increased or decreased) by $\omega$. As a result, not all terms on the right-hand side of (7) represent the correction to the direct amplitude $\mathcal{M}_{0}$, which implies the validity of (6). For example, evaluating $\mathcal{M}_{1}$ (the second diagram in figure 1) one finds, that it contains the terms proportional to $\delta\left(\varepsilon-\varepsilon_{\mathrm{i}}\right)$ and to $\delta\left(\varepsilon-\varepsilon_{\mathrm{i}}-2 \omega\right)$, which conflict with the conservation law (6). Therefore, the term $\mathcal{M}_{1}$ must be ignored when constructing the correction to $\mathcal{M}_{0}$.

The lowest-order correction originates from the amplitude $\mathcal{M}_{2}$ (the last diagram in figure 1), which contains the terms proportional to $\delta\left(\varepsilon-\varepsilon_{\mathrm{i}}-\omega\right)$. Applying the 
standard technique of the time-dependent perturbation theory, one derives the following expression for this contribution:

$$
\left.\mathcal{M}_{2}\right|_{\varepsilon=\varepsilon_{\mathrm{i}}+\omega}=2 \pi \delta\left(\varepsilon-\varepsilon_{\mathrm{i}}-\omega\right) M_{2}
$$

where

$$
\begin{aligned}
M_{2} & =\frac{|\alpha(\omega)|^{2} E_{0}^{2}}{8} \iint \mathrm{d} \mathbf{r}_{1} \mathrm{~d} \mathbf{r}_{2} \psi_{\mathbf{p}}^{(-) *}\left(\mathbf{r}_{2}\right) \frac{\mathbf{e}_{0} \mathbf{r}_{2}}{r_{2}^{3}}\left(G_{\varepsilon_{\mathrm{i}}}\left(\mathbf{r}_{2}, \mathbf{r}_{1}\right)+G_{\varepsilon+\omega}\left(\mathbf{r}_{2}, \mathbf{r}_{1}\right)\right) \frac{\mathbf{e}_{0} \mathbf{r}_{1}}{r_{1}^{3}} \\
& \times \int \mathrm{d} \mathbf{r} G_{\varepsilon}\left(\mathbf{r}_{1}, \mathbf{r}\right)\left(\mathbf{E}_{0} \mathbf{r}\right) \psi_{i}(\mathbf{r}) .
\end{aligned}
$$

Here $G_{E}\left(\mathbf{r}^{\prime}, \mathbf{r}\right)$ is the Green's function of the photoelectron and $\mathbf{e}_{0}=\mathbf{E}_{0} / E_{0}$ is the unit vector of the laser field polarization.

Hence, within the second second order perturbation theory in $\mathcal{U}(\mathbf{r}, t)$, the photoionization amplitude is written as:

$$
\left.\mathcal{M}_{2}\right|_{\varepsilon=\varepsilon_{\mathrm{i}}+\omega} \approx 2 \pi \delta\left(\varepsilon-\varepsilon_{\mathrm{i}}-\omega\right)\left(M_{0}+M_{2}\right)
$$

Using the amplitude (12) one can write the following expression for the spectraangular distribution:

$$
\frac{\mathrm{d} \sigma}{\mathrm{d} \Omega}=\frac{p}{4 \pi^{2} c}\left|M_{0}+M_{2}\right|^{2} \approx \frac{\mathrm{d} \sigma_{0}}{\mathrm{~d} \Omega}(1+K(\omega, \theta)) .
$$

Here

$$
\frac{\mathrm{d} \sigma_{0}}{\mathrm{~d} \Omega}=\frac{p}{4 \pi^{2} c}\left|M_{0}\right|^{2}
$$

stands for the differential cross section calculated without the influence of the induced dipole moment. Within the dipole-photon approximation the differential cross section $\mathrm{d} \sigma_{0} / \mathrm{d} \Omega$ can be presented in the well-known general form (see, e.g., [6]):

$$
\frac{\mathrm{d} \sigma_{0}}{\mathrm{~d} \Omega}=\frac{\sigma_{0}}{4 \pi}\left(1+\beta(\omega) P_{2}(\cos \theta)\right)
$$

where $\sigma_{0} \equiv \sigma_{0}(\omega)$ is the total cross section of photoionization, $\theta$ stands for the angle between $\mathbf{p}$ and $\mathbf{e}_{0}, P_{2}(\cos \theta)$ is the Legendre polynomial of the second order. The quantity $\beta(\omega)$ stands for the angular asymmetry parameter [7]. The factor $1+\beta(\omega) P_{2}(\cos \theta)$ defines the profile of the angular distribution.

Equation (13) indicates, that the profile is modified by the factor $1+K(\omega, \theta)$, when the field (4) is taken into account. The correction term $K(\omega, \theta)$ is defined as follows:

$$
K(\omega, \theta)=2 \operatorname{Re} \frac{M_{2}}{M_{0}} .
$$

Formula (13) was obtained by means of the perturbation theory. Thus, it is implied that the absolute value of the correction term satisfies the condition $|K(\omega, \theta)|<1$. If otherwise, the field $\mathcal{U}(\mathbf{r}, t)$ must be accounted for in all orders of the perturbation series (7).

It follows from $(11)$, that $K(\omega, \theta)$ is proportional to the squared induced dipole moment, $K \propto|\mathbf{d}|^{2}=|\alpha(\omega)|^{2} E_{0}^{2}$. Therefore, for a target of a small polarizability, one has to apply very intensive laser fields in order to achieve a noticeable correction 
to the photoelectron angular distribution, i.e. $|K(\omega, \theta)| \sim 1$. On the contrary, for a strongly polarizable target (e.g., a metallic cluster or/and its anion) a strong effect can be expected for laser fields of a moderate strength. In section 3 we demonstrate, that dramatic change of the angular distribution profile can occur for $E_{0} \sim 10^{6} \mathrm{~V} / \mathrm{cm}$.

It is important to note, that the correction term (16) depends on the ratio of the amplitudes $M_{2}$ and $M_{0}$. Thus, one can expect that it is less sensitive to the approximation chosen to describe the photoelectron wavefunction than the cross section $\mathrm{d} \sigma_{0} / \mathrm{d} \Omega$. Therefore, to estimate the influence of the target polarization one can calculate $K(\omega, \theta)$ within the framework of a comparatively simple approximation, which is described below.

\subsection{Analytic expression for $K(\omega, \theta)$}

In this section we derive an approximate expression for the correction term $K(\omega, \theta)$ using a simple, although realistic and physically clear, model.

To start with let us assume, that the ionized target is a spherically-symmetric anion. Then, firstly, the ground state wavefunction $\psi_{i}(\mathbf{r})$ does not depend on the angles of the position vector: $\psi_{i}(\mathbf{r})=\psi_{i}(r)$. Secondly, the wavefunction $\psi_{\mathbf{p}}^{(-)}(\mathbf{r})$ of the photoelectron does not contain the contribution of a long-range Coulomb field of the residue. These assumptions, being not of a principal nature, allow one to simplify the intermediate algebra.

Making use of the partial wave expansion

$$
\psi_{\mathbf{p}}^{(-)}(\mathbf{r})=\frac{4 \pi}{p r} \sum_{l m} \mathrm{i}^{l} \mathrm{e}^{-\mathrm{i} \delta_{l}(p)} Y_{l m}^{*}\left(\mathbf{n}_{\mathbf{r}}\right) Y_{l m}\left(\mathbf{n}_{\mathbf{p}}\right) \chi_{p l}(r),
$$

where $Y_{l m}(\mathbf{n})$ are the spherical harmonics, $\delta_{l}(p)$ are the scattering phaseshift, and $\chi_{p l}(r)$ are the radial wavefunctions, one writes the amplitude $M_{0}$ in the following form:

$$
M_{0}=-\left.2 \pi \mathrm{i} \frac{\mathbf{E}_{0} \mathbf{p}}{p^{2}} \mathrm{e}^{\mathrm{i} \delta_{l}(p)} \int_{0}^{\infty} \mathrm{d} r r^{2} \chi_{p l}(r) \psi_{i}(r)\right|_{l=1} .
$$

Another simplification is based on the approximation that the field $\mathcal{U}(\mathbf{r}, t)$ acts on the escaping electron only outside the target, i.e. at the distances $r>R$. Additionally, one can assume, that the wavefunction $\psi_{i}(r)$ is concentrated in the interior of the target, i.e. at $r<R$. Therefore, when evaluating the right-hand side of (11) one takes into account that $r_{1}, r_{2}>R>r$. This allows one to relate $M_{2}$ to the amplitude $M_{0}$. To do this let us approximate the Green's function $G_{\varepsilon}\left(\mathbf{r}_{1}, \mathbf{r}\right)$ as follows:

$G_{\varepsilon}\left(\mathbf{r}_{1}, \mathbf{r}\right)=2 \mathrm{i} p \sum_{l m} \mathcal{G}_{\varepsilon l}\left(r_{1}, r\right) Y_{l m}\left(\mathbf{n}_{1}\right) Y_{l m}^{*}(\mathbf{n}) \approx \frac{2 \mathrm{i}}{p} \sum_{l m} \frac{X_{p l}\left(r_{1}\right) \chi_{p l}(r)}{r_{1} r} Y_{l m}\left(\mathbf{n}_{1}\right) Y_{l m}^{*}(\mathbf{n})$.

Here $r_{>} / r_{<}$is the largest/smallest of $r_{1}, r, X_{p l}(r)$ stands for the irregular solutions of the radial Schrödinger equation for the energy $\varepsilon=p^{2} / 2$. The quantity $g_{\varepsilon l}\left(r_{1}, r\right)=$ $X_{p l}\left(r_{>}\right) \chi_{p l}\left(r_{<}\right) / p^{2} r_{>} r_{<}$is the exact radial Green's function corresponding to the multipolarity $l$. The first equality in (19) states the partial-wave expansion of the Green's function. The approximate equality is based on the assumption $r_{1}>r$. 
Using (19) in (11) one notices that the radial integral over $r$ reduces to that from (18). Therefore, the amplitude $M_{2}$ acquires the form:

$$
M_{2} \approx-\gamma(\omega, \theta) M_{0}
$$

with

$$
\begin{aligned}
\gamma(\omega, \theta) & =|\alpha(\omega)|^{2} E_{0}^{2} \frac{\mathrm{e}^{-\mathrm{i} \delta_{1}(p)}}{8 \pi} \cos ^{-1} \theta \\
& \times \iint \mathrm{d} \mathbf{r}_{1} \mathrm{~d} \mathbf{r}_{2} \psi_{\mathbf{p}}^{(-) *}\left(\mathbf{r}_{2}\right) \frac{\mathbf{e}_{0} \mathbf{n}_{2}}{r_{2}^{2}}\left(G_{\varepsilon_{\mathrm{i}}}\left(\mathbf{r}_{2}, \mathbf{r}_{1}\right)+G_{\varepsilon+\omega}\left(\mathbf{r}_{2}, \mathbf{r}_{1}\right)\right) \frac{\left(\mathbf{e}_{0} \mathbf{n}_{1}\right)^{2}}{r_{1}^{3}} X_{p 1}\left(r_{1}\right) .
\end{aligned}
$$

Here the integration is carried out over the spatial region $r_{1}, r_{2}>R$.

The correction term (16) is related to $\gamma(\omega, \theta)$ as $K(\omega, \theta)=-2 \operatorname{Re} \gamma(\omega, \theta)$.

Let us comment on the evaluation of the right-hand side of (21). Introducing the expansion (17) and representing the Green's functions $G_{\varepsilon_{\mathrm{i}}}\left(\mathbf{r}_{2}, \mathbf{r}_{1}\right)$ and $G_{\varepsilon+\omega}\left(\mathbf{r}_{2}, \mathbf{r}_{1}\right)$ in terms of the partial-wave series similar to (19), one carries out the angular integration by means of the standard methods (see, e.g., [8]) and arrives at:

$$
\begin{aligned}
K(\omega, \theta) & =-2 \operatorname{Re} \gamma(\omega, \theta) \\
& =-\frac{1}{3}|\alpha(\omega)|^{2} E_{0}^{2} \sum_{l l^{\prime}}\left[\delta_{l 1}\left(\delta_{l^{\prime} 0}+\frac{4}{5} \delta_{l^{\prime} 2}\right)-\frac{6}{5} \delta_{l 3} \delta_{l^{\prime} 2} \frac{P_{3}(\cos \theta)}{\cos \theta}\right] \operatorname{Im} B_{l l^{\prime}} .
\end{aligned}
$$

The Kronecker symbols $\delta_{i j}$, which reduce the allowed values of the orbital momenta to $l=1,3$ and $l^{\prime}=0,2$, reflect the dipole selection rules applicable to each of the vertices in the last diagram in figure 1 . The ratio $P_{3}(\cos \theta) / \cos \theta$ reduces to $\left(5 P_{2}(\cos \theta)-2\right) / 3$ with the help of the recurrence relation for the Legendre polynomials.

The quantities $B_{l l^{\prime}}$ stand for the following radial integrals:

$$
B_{l l^{\prime}}=\frac{\mathrm{e}^{\mathrm{i}\left(\delta_{l}(p)-\delta_{1}(p)\right)}}{p} \int_{R}^{\infty} \int_{R}^{\infty} \mathrm{d} r_{1} \mathrm{~d} r_{2} \frac{X_{p 1}\left(r_{1}\right)}{r_{1}} \frac{\chi_{p l}\left(r_{2}\right)}{r_{2}}\left(g_{\varepsilon_{\mathrm{i}} l^{\prime}}\left(r_{2}, r_{1}\right)+g_{\varepsilon+\omega l^{\prime}}\left(r_{2}, r_{1}\right)\right) \text {. }
$$

For any realistic target the exact evaluation of these integrals can be done by numerical means only, implying the computation of the regular $\chi_{p l}(r)$ and the irregular $X_{p 1}(r)$ functions as well as of the Green's functions $g_{\varepsilon_{\mathrm{i}} l^{\prime}}\left(r_{2}, r_{1}\right)$ and $g_{\varepsilon+\omega l^{\prime}}\left(r_{2}, r_{1}\right)$. To carry out the approximate integration one can substitute these quantities with the leading terms of their asymptotic representations. For $\chi_{p l}(r)$ and $X_{p 1}(r)$ this results in $\chi_{p l}(r) \sim \sin \left(p r-\pi l / 2+\delta_{l}(p)\right)$ and $X_{p 1}(r) \sim-\mathrm{e}^{\mathrm{i}\left(p r+\delta_{1}(p)\right)}$. The leading terms in the expansions of the radial Green's functions are:

$$
\left\{\begin{array}{l}
g_{\varepsilon+\omega l^{\prime}}\left(r_{2}, r_{1}\right) \sim-\frac{\mathrm{i}}{P} \frac{\mathrm{e}^{\mathrm{i} P r_{>}}}{r_{>}} \frac{S_{l^{\prime}}(P) \mathrm{e}^{\mathrm{i} P r_{<}}-\mathrm{e}^{-\mathrm{i} P r_{<}}}{r_{<}}, \\
g_{\varepsilon_{\mathrm{i}} l^{\prime}}\left(r_{2}, r_{1}\right) \sim-\frac{1}{p_{i}} \frac{\mathrm{e}^{-p_{i} r_{>}}}{r_{>}} \frac{S_{l^{\prime}}\left(\mathrm{i} p_{i}\right) \mathrm{e}^{-p_{i} r_{<}}-\mathrm{e}^{p_{i} r_{<}}}{r_{<}}
\end{array}\right.
$$

Here $P=\sqrt{2(\varepsilon+\omega)}, p_{i}=\sqrt{-2 \varepsilon_{\mathrm{i}}}, S_{l^{\prime}}(P)$ and $S_{l^{\prime}}\left(\mathrm{i} p_{i}\right)$ are the elements of the scattering matrix. For the real argument $P$ this quantity reads as $S_{l^{\prime}}(P)=\mathrm{e}^{2 \mathrm{i} \delta_{l^{\prime}}(P)}$.

By using the asymptotic formulae one carries out analytical evaluation of the integrals from (23). The final result (the leading term) for $\operatorname{Im} B_{l l^{\prime}}$ is as follows:

$\operatorname{Im} B_{l l^{\prime}}=\frac{(-1)^{(l-1) / 2}}{2 X^{4}}\left[\xi^{-1} \frac{\xi^{2}+1}{\left(\xi^{2}-1\right)^{2}}\right.$ 


$$
\begin{aligned}
& +\xi^{-1} \operatorname{Re}\left(\frac{S_{l^{\prime}}(P) S_{l}(p) \mathrm{e}^{2 \mathrm{i}(\xi+1) X}}{(\xi+1)^{2}}+\frac{S_{l^{\prime}}(P) \mathrm{e}^{2 \mathrm{i} \xi X}}{\xi^{2}-1}-\frac{S_{l}(p) \mathrm{e}^{2 \mathrm{i} X}}{\xi+1}\right) \\
& \left.+\xi_{i}^{-1} \operatorname{Im} \frac{S_{l}(p)\left(\mathrm{i} \xi_{i}-1\right) \mathrm{e}^{2 \mathrm{i} X}}{\left(\xi_{i}^{2}+1\right)}\right]_{\substack{l=1,3 \\
l^{\prime}=0,2}},
\end{aligned}
$$

where $X=p R, \xi=P / p$ and $\xi_{i}=p_{i} / p$. It follows from the approximations formulated above, that formula (25) is valid if the photoelectron momentum $p$ and the ground state energy $\varepsilon_{\mathrm{i}}$ satisfy the conditions:

$$
p R>1, \quad \sqrt{-2 \varepsilon_{\mathrm{i}}} R>1 .
$$

Letting $\delta_{l}(p)=\delta_{l^{\prime}}(P) \equiv 0$ in (25) one finds the plane-wave Born limit of $\operatorname{Im} B_{l l^{\prime}}$.

The presence of oscillatory terms on the right-hand side of (25) is physically clear. Indeed, in the limit $p R>1$ the wavelength of the photoelectron is less than the size of the target, which is supposed to have a well-pronounced edge (e.g., the edge of an ionic core in a cluster). Thus, the electron experiences a diffraction at the edge when leaving the core. This diffraction leads to the oscillatory character of the cross section. This phenomenon was discussed previously in connection with various processes involving metallic clusters and fullerenes: the radiative electron capture by metallic clusters [9], the electron scattering [10], the polarizational bremsstrahlung [11].

Carrying out obvious summations in (22), one writes correction factor as follows:

$$
K(\omega, \theta)=E_{0}^{2}\left(\kappa_{0}(\omega)+\kappa_{2}(\omega) P_{2}(\cos \theta)\right),
$$

where

$$
\kappa_{0}(\omega)=-\frac{1}{3}|\alpha(\omega)|^{2} \operatorname{Im}\left(B_{10}+\frac{4}{5} B_{12}+\frac{4}{5} B_{32}\right), \quad \kappa_{2}(\omega)=\frac{2}{3}|\alpha(\omega)|^{2} \operatorname{Im} B_{32} .
$$

Formula (27) stresses the proportionality of $K(\omega, \theta)$ to the laser field intensity (which is proportional to $E_{0}^{2}$ ) and exhibits explicitly its dependence on the emission angle $\theta$, which enters, as well as in (15), via the second order Legendre polynomial.

As a function of $\theta$ the modulus of $K(\omega, \theta)$ attains its maximum either at $\theta=0^{\circ}$ (and $\theta=180^{\circ}$ ) or at $\theta=90^{\circ}$, depending on the sign of the product $\kappa_{0}(\omega) \kappa_{2}(\omega)$ and on the relative magnitudes of $\kappa_{0}(\omega)$ and $\kappa_{2}(\omega)$. For further reference let us define the quantity

$$
\begin{aligned}
|\kappa(\omega)|_{\max } & \equiv\left|\kappa_{0}(\omega)+\kappa_{2}(\omega) P_{2}(\cos \theta)\right|_{\max } \\
& = \begin{cases}\left|\kappa_{0}(\omega)+\kappa_{2}(\omega)\right| & \text { if } \kappa_{0}(\omega) \kappa_{2}(\omega)>0, \\
\left|\kappa_{0}(\omega)\right|+\left|\kappa_{2}(\omega)\right| / 2 & \text { if } \kappa_{0}(\omega) \kappa_{2}(\omega)<0 \text { and } 4\left|\kappa_{0}(\omega)\right|>\left|\kappa_{2}(\omega)\right|, \\
\left|\kappa_{2}(\omega)\right|-\left|\kappa_{0}(\omega)\right| & \text { if } \kappa_{0}(\omega) \kappa_{2}(\omega)<0 \text { and } 4\left|\kappa_{0}(\omega)\right|<\left|\kappa_{2}(\omega)\right| .\end{cases}
\end{aligned}
$$

which allows one to estimate the laser field strength needed to achieve a noticeable correction to the angular distribution (see the discussion in section 3).

Substituting (27) into (13), taking into account (15) and expressing $P_{2}^{2}(\cos \theta)$ via $P_{2}(\cos \theta)$ and $P_{4}(\cos \theta)$, one derives the following formula for the differential cross section within the lowest order of perturbation theory in $\mathcal{U}(\mathbf{r}, t)$ :

$$
\frac{\mathrm{d} \sigma}{\mathrm{d} \Omega}=\frac{\sigma_{0}}{4 \pi}\left(a_{0}(\omega)+a_{2}(\omega) P_{2}(\cos \theta)+a_{4}(\omega) P_{4}(\cos \theta)\right) .
$$


The coefficients $a_{0,2,4}$ are related to the asymmetry parameter $\beta$ and to $\kappa_{0,2}$ via

$a_{0}=1+E_{0}^{2}\left(\kappa_{0}+\frac{\beta \kappa_{2}}{5}\right), \quad a_{2}=\beta+E_{0}^{2}\left[\kappa_{2}+\left(\kappa_{0}+\frac{2 \kappa_{2}}{7}\right) \beta\right], \quad a_{4}=\frac{18}{35} E_{0}^{2} \beta \kappa_{2} .(31)$

The right-hand side of (30) suggests that not only the profile of the angular distribution can be changed, but the magnitude of the cross section, integrated over the emission angles, is scaled by the factor $a_{0}$ which can be greater or lower than one depending on the signs and magnitudes of $\beta$ and $\kappa_{0,2}$.

If the action of $\mathcal{U}(\mathbf{r}, t)$ is ignored then $a_{0} \rightarrow 1, a_{0} \rightarrow \beta$ and $a_{4} \rightarrow 0$, and equation (30) reduces to (15).

\section{Numerical results}

Numerical analysis of the influence of the induced dipole moment on the profile of the angular distribution was carried out for the process of photodetachment of sodium cluster anions $\mathrm{Na}_{N}^{-}$. The results presented below in this section refer to $N=8,20,40,58$ and 92. The corresponding neutral clusters are spherically-symmetric which makes applicable the theory described in section 2 .

The parameters of sodium clusters and their anions, used in our numerical calculations, are summarized in table 1. Let us comment on these data.

The ionic core radius was calculated using the standard relation $R=r_{s} N^{1 / 3}$ with the Wigner-Seitz radius $r_{s}$ set to 4 a.u. The values of electron affinities $I_{\mathrm{a}}$ are taken from a recent work by Kostko [5] which contains, in particular, an excellent collection of reference data on various metal clusters. The other data presented in the table refer to the polarizability of the core and the parameters of the plasmon peak. We considered the dynamic dipole polarizability $\alpha(\omega)$ of the clusters within the framework of the resonance plasmon approximation (see, e.g., $[11,12])$ :

$$
\alpha(\omega)=\alpha(0) \frac{\omega_{\mathrm{s}}^{2}}{\omega_{\mathrm{s}}^{2}-\omega^{2}-\mathrm{i} \omega \Gamma} .
$$

Here $\alpha(0)$ is the static polarizability, $\omega_{\mathrm{s}}$ is the surface plasmon energy, and $\Gamma$ is the linewidth of the plasmon resonance.

Due to the spill-out effect the static polarizability of a metallic cluster of a radius $R$ exceeds the classical value $\alpha_{\mathrm{cl}}=R^{3}$, which characterizes a metallic ball of the same radius. In the table we present the ratio $\alpha(0) / \alpha_{\mathrm{cl}}$ calculated within the RPA [13] and the LDA [14]) as well as the experimentally measured values [15]. The $\omega_{\mathrm{s}}$ values were obtained with the help of the relation proposed in [16] to account for the size-dependence of the plasmon energy: $\omega_{\mathrm{s}}=\omega_{\mathrm{Mie}}(1-1.5 \delta / R)$, where $\omega_{\text {Mie }}=3.27 \mathrm{eV}$ is the Mie plasmon energy in a sodium metallic sphere, and $\delta=0.54 \AA$ takes into account the spill-out effect.

The size dependence of the width $\Gamma$ of the plasmon resonance for neutral sodium clusters with $N$ up to several hundreds was analyzed in [17] within the RPA scheme and in [18] within a semiclassical approach. These two methods, being consistent for $N>58$, 
Table 1. Data on several spherically-symmetric sodium clusters: the ionic core radius $R$; the electron affinity $I_{\mathrm{a}}[5], \alpha_{\mathrm{cl}}=R^{3}$ is the classical static polarizability, $\alpha(0)$ stands for calculated $[13,14]$ and measured [15] static polarizability; $\omega_{\mathrm{s}}$ is the surface plasmon energy calculated according to [16], $\Gamma$ is the width of the plasmon resonance peak [18].

\begin{tabular}{|c|c|c|c|c|c|c|c|c|}
\hline & $\begin{array}{c}R \\
\text { (a.u.) }\end{array}$ & $\begin{array}{c}I_{\mathrm{a}} \\
(\mathrm{eV})\end{array}$ & $\begin{array}{c}\alpha_{\mathrm{cl}} \\
\text { (a.u.) }\end{array}$ & [13] & $\begin{array}{c}(0) / \alpha_{c} \\
{[14]}\end{array}$ & {$[15]$} & $\begin{array}{l}\omega_{\mathrm{s}} \\
(\mathrm{eV})\end{array}$ & $\begin{array}{c}\Gamma \\
(\mathrm{eV})\end{array}$ \\
\hline $\mathrm{Na}_{8}$ & 8.00 & 0.915 & 512 & 1.48 & 1.44 & 1.72 & 2.64 & 0.2 \\
\hline $\mathrm{Na}_{20}$ & 10.86 & 1.337 & 1280 & 1.41 & 1.37 & 1.67 & 2.81 & 0.4 \\
\hline $\mathrm{Na}_{40}$ & 13.68 & 1.509 & 2560 & 1.10 & 1.32 & 1.60 & 2.90 & 0.7 \\
\hline $\mathrm{Na}_{58}$ & 15.48 & 1.719 & 3712 & 1.24 & 1.22 & - & 2.95 & 0.4 \\
\hline $\mathrm{Na}_{92}$ & 18.06 & 1.847 & 5888 & 1.22 & 1.20 & - & 2.99 & 0.2 \\
\hline
\end{tabular}

deviate noticeably for lower $N$ values. The RPA calculations reproduce the $1 / R$ scaling law which, is inadequate when compared to the experimental data for $N \lesssim 58$ (see the discussion in [17]). The semiclassical calculations [18] seem to be consistent with the experimental data in the whole region $N=10 \ldots 100$. The values of $\Gamma$, presented in the table, were deduced from figure 1 in [18].

It is seen from the table, that for all $N$ the plasmon energy exceeds the electron affinity. This fact justifies the choice of cluster anions in a view of the present study. Indeed, the inequality $\omega_{\mathrm{s}}>I_{\mathrm{a}}$ leads to the additional enhancement of $K(\omega, \theta)$ owing to the pronounced increase of the modulus of the dynamic polarizability in the region $\omega \approx \omega_{\mathrm{s}}$.

To conclude the discussion on the parameters of the chosen targets let us note, that both criteria from (26) can be easily met for $\mathrm{Na}_{N}^{-}$. The first inequality fails only in the narrow near-threshold region $\omega-I_{\mathrm{a}} \ll I_{\mathrm{a}}$ but is well fulfilled for $\omega \approx \omega_{\mathrm{s}}$. The validity of the second condition one proves by introducing $\varepsilon_{\mathrm{i}}=-I_{\mathrm{a}}$.

The results of calculation of the quantities relevant to the correction term $K(\omega, \theta)$ are presented in figures 2 and 3. For each anion the calculations were performed for the photon energies above the ionization threshold $I_{\mathrm{a}}$ and in the vicinity of the plasmon peak. The latter manifests itself as a resonance in the dependence of $|\alpha(\omega)|^{2}$ on $\omega$, as it is seen from the upper panel in each graph. The dynamic polarizabilities were calculated via (32) with the parameters $\omega_{\mathrm{s}}$ and $\Gamma$ as indicated in table 1. Given the differences between the calculated and experimentally measured values of the static polarizabilities $\alpha(0)$, as well as the absence of the experimental data for $\mathrm{Na}_{58}$ and $\mathrm{Na}_{92}$, for the ratio $\alpha(0) / R^{3}$ we used the values close to the theoretical data (see the figures captions).

The dependences of the coefficients $\kappa_{0}(\omega)$ and $\kappa_{2}(\omega)$ on $\omega$ are presented in the middle panels. They were computed from (28) and (25). To calculate the right-hand side of the latter formula beyond the plane-wave Born approximation one must know the scattering phaseshifts $\delta_{l}(p)$ and $\delta_{l}(P)$. To determine the phaseshifts we solved numerically the phase equation (see, e.g., $[19,20]$ ) for an electron moving in a local potential. The model potential, which was used to describe the interaction between the photoelectron and the neutral residue, accounted for the following three terms: (a) 

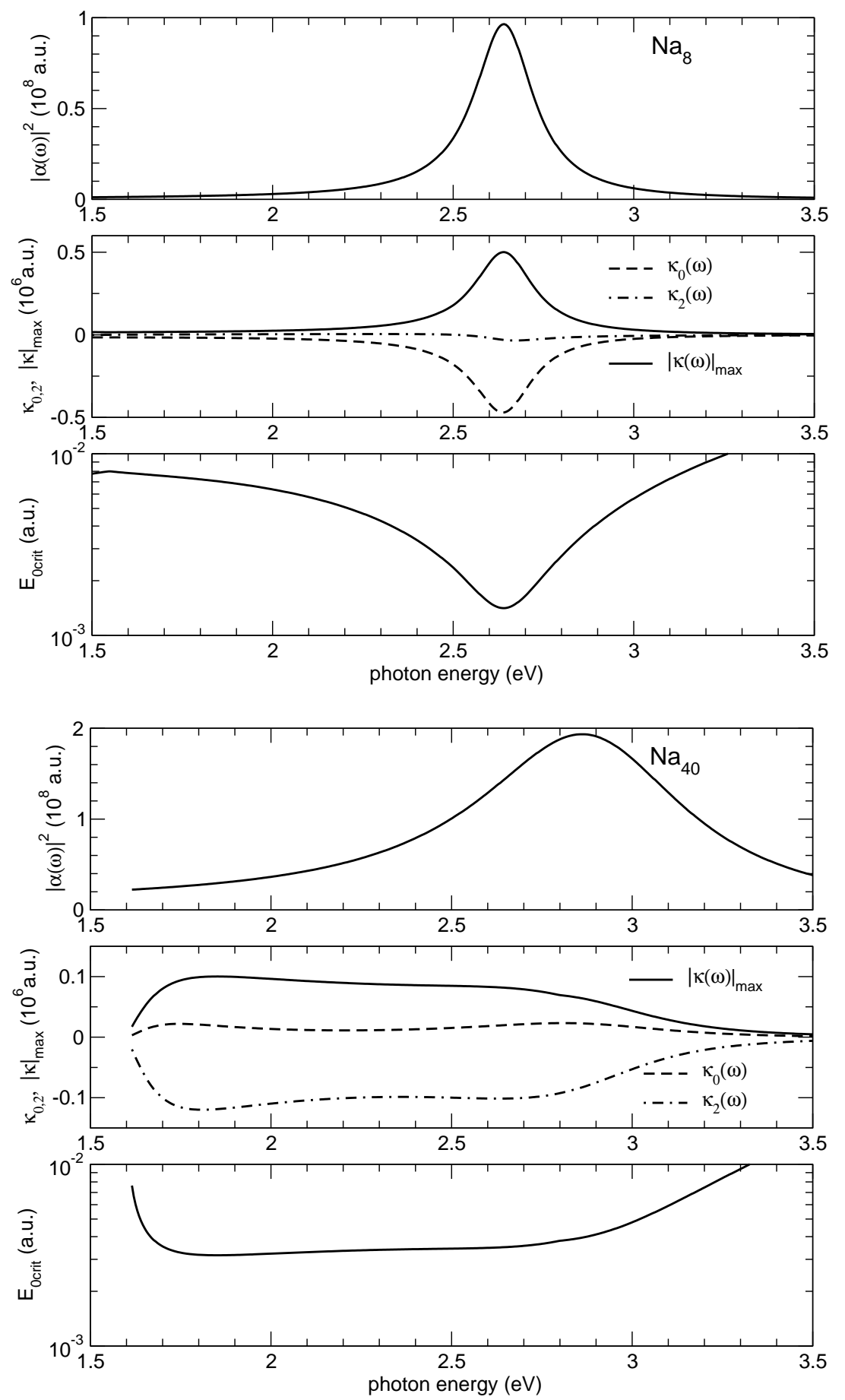

Figure 2. Calculation of the correction term for $\mathrm{Na}_{8}^{-}$and for $\mathrm{Na}_{40}^{-}$as indicated. In each graph the upper panel represents the dependence of $|\alpha(\omega)|^{2}$ on $\omega$; the middle panel - dependences $\kappa_{0,2}(\omega)$ and $|\kappa(\omega)|_{\max }$, eqs. (28)-(29); the lower panel - dependence of $E_{0 \text { crit }}(\omega)$, eq. (33). Parameters $\omega_{\mathrm{s}}$ and $\Gamma$ are as indicated in table 1. The data refer to the $\alpha(0) / R^{3}$ ratio equal to 1.45 for $\mathrm{Na}_{8}$ and to 1.30 for $\mathrm{Na}_{40}$.

the short-range potential due to the Coulomb interaction with the core's electrons and the ionic jellium core, (b) the exchange-correlation term, which was treated within the 

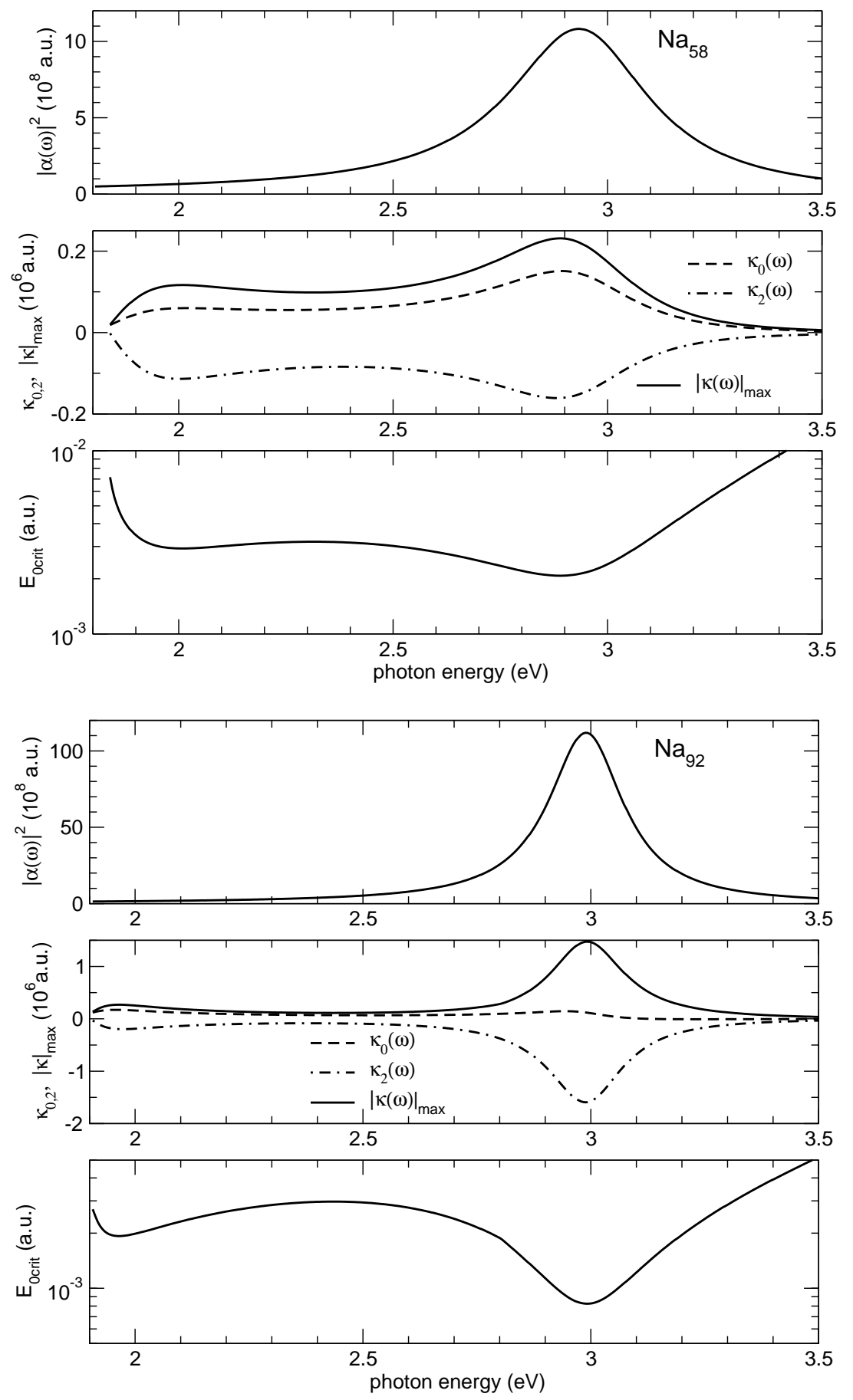

Figure 3. Same as in figure 2 but for $\mathrm{Na}_{58}^{-}$and $\mathrm{Na}_{92}^{-}$. For both clusters the ratio $\alpha(0) / R^{3}$ is set to 1.20 .

Gunnarsson-Lundqvist approximation [21], (c) the polarization potential was considered in the form $U_{\mathrm{pol}}(r)=-\alpha(0) / 2\left(r^{2}+R^{2}\right)^{2}$ for $r>R$ and $U_{\mathrm{pol}}(r)=0$ if otherwise. Inclusion of the polarization term is important for an adequate description the low-energy electron scattering from metallic clusters (e.g., [22]). A more rigorous treatment accounts for the many-body correlations, which may be considered, for example, within the Dyson 
equation scheme [23]. However, for the purpose of our paper it is sufficient to treat the polarization potential in the simple form described above.

Comparing the middle panels in the graphs from figures 2 and 3 one notices that the behaviour of $\kappa_{0,2}(\omega)$ (and of $|\kappa(\omega)|_{\max }$ as well) exhibits some general trends along with the specific features determined by the dynamic and geometrical properties of an individual target. Common to all clusters is the existence of extrema for $\kappa_{0,2}(\omega)$ at $\omega \approx \omega_{\mathrm{s}}$, which is due to the factor $|\alpha(\omega)|^{2}$, see (28). The extremum can be clearly defined (see the curves $\kappa_{0}(\omega)$ for $\mathrm{Na}_{8}$ and $\kappa_{2}(\omega)$ for $\mathrm{Na}_{92}$ which have distinct maxima) or less pronounced (both curves for $\mathrm{Na}_{58}$ ) or barely seen (the case of $\mathrm{Na}_{40}$ ). The variation in the shape of the extremum, as well as the behaviour of the curves in the regions below and above the plasmon resonance, can be understood by analyzing the $\omega$-dependence of the right-hand sides in (28) and (25). The non-oscillatory part of the latter depends on the photon energy mainly through the factor $(p R)^{-4}=4 R^{-4}\left(\omega-I_{\mathrm{a}}\right)^{-2}$. This leads to $\kappa_{0,2}(\omega) \propto|\alpha(\omega)|^{2} /\left(\omega-I_{\mathrm{a}}\right)^{2}$. Therefore, a symmetric resonance profile of $|\alpha(\omega)|^{2}$ is distorted due to the factor $\left(\omega-I_{\mathrm{a}}\right)^{-2}$. For a powerful and narrow resonance (as in the case of $\mathrm{Na}_{92}$ ) the distortion is comparatively small, whereas for a wide resonance the disproportion between the low- and the high- $\omega$ shoulders may completely change the behaviour of the curve in the vicinity of the resonance, as it happens for $\mathrm{Na}_{40}$. In either case the factor $\left(\omega-I_{\mathrm{a}}\right)^{-2}$ results in a faster decrease of $\left|\kappa_{0,2}(\omega)\right|$ with $\omega$ beyond the resonance. In the region $I_{\mathrm{a}}<\omega<\omega_{\mathrm{s}}$ the curves $\kappa_{0,2}(\omega)$, being enhanced by the factor, exhibit a non-monotonous oscillatory character (most clearly seen in $\mathrm{Na}_{40}, \mathrm{Na}_{58}$ and $\mathrm{Na}_{92}$ graphs). This is due to the second and the third terms on the right-hand side of (25), which contain the oscillating factors $\exp (\mathrm{i} p R)$ and $\exp (\mathrm{i} P R)$, dependent on the cluster size, and the factors $S_{l}(p)=\exp \left(\mathrm{i} \delta_{l}(p)\right), S_{l^{\prime}}(P)=\exp \left(\mathrm{i} \delta_{l}(P)\right)$, dependent on the phaseshifts.

The solid curves in the middle panels of each graph represent the dependences $|\kappa(\omega)|_{\max }$ defined in (29). For a given photon energy the quantity $E_{0}^{2}|\kappa(\omega)|_{\max }$ stands for the maximum value of $|K(\omega, \theta)|$ within the interval $\theta=\left[0^{\circ}, 180^{\circ}\right]$. It was already mentioned, that the correction term must satisfy the condition $|K(\omega, \theta)|<1$. Thus, taking into account that $|K(\omega, \theta)| \leq E_{0}^{2}|\kappa(\omega)|$, one can introduce a critical value $E_{0 \text { crit }}(\omega)$, which defines the upper boundary for the laser field strength consistent with the perturbative approach adopted in this paper:

$$
E_{0 \text { crit }}(\omega)=\left(|\kappa(\omega)|_{\max }\right)^{-1 / 2} .
$$

The corresponding intensity of the laser field (in $\mathrm{W} / \mathrm{cm}^{2}$ ) can be calculated from the relation $I_{0 \text { crit }} \approx 3.3 \times 10^{16} E_{0 \text { crit }}^{2}$, where $E_{0 \text { crit }}$ is measured the atomic units.

In the limit $E_{0} \ll E_{0 \text { crit }}(\omega)$ a strong inequality $|K(\omega, \theta)| \ll 1$ is valid, so that the photoionization process is not affected by the induced dipole moment. The influence of the latter can become pronounced for $E_{0} \lesssim E_{0 \text { crit }}(\omega)$ resulting in $|K(\omega, \theta)| \sim 1$.

Dependences $E_{0 \text { crit }}(\omega)$ are plotted in the lower panels in figures 2 and 3. For all clusters this function attains its minimum in the point $\tilde{\omega} \approx \omega_{\mathrm{s}}$ in the vicinity of the plasmon resonance. The values of $\tilde{\omega}$, as well as of other quantities calculated in this 
Table 2. The minima $\tilde{\omega}$ of the functions $E_{0 \text { crit }}(\omega)$ (bottom panels in the graphs in figures 2 and 3 ), the values of $\kappa_{0,2}$ and $|\kappa|_{\max }$ in these points, the critical strength of the laser field $E_{0 \text { crit }}(\tilde{\omega})=\left(|\kappa(\tilde{\omega})|_{\max }\right)^{-1 / 2}$ and the corresponding intensity $I_{0 \text { crit }}(\tilde{\omega})$, the ponderomotive energy $E_{0 \text { crit }}^{2}(\tilde{\omega}) / 4 \tilde{\omega}^{2} ; E_{\mathrm{a}}$ is the internal anionic field.

\begin{tabular}{l|crrccccc}
\hline & $\begin{array}{c}\tilde{\omega} \\
(\mathrm{eV})\end{array}$ & $\begin{array}{r}\kappa_{0}(\tilde{\omega}) \\
\left(10^{6}\right)\end{array}$ & $\begin{array}{r}\kappa_{2}(\tilde{\omega}) \\
\left(10^{6}\right)\end{array}$ & $\begin{array}{c}|\kappa(\tilde{\omega})|_{\max } \\
\left(10^{6}\right)\end{array}$ & $\begin{array}{c}E_{\text {0crit }}(\tilde{\omega}) \\
\left(10^{-3} \text { a.u. }\right)\end{array}$ & $\begin{array}{c}I_{\text {0crit }}(\tilde{\omega}) \\
\left(10^{11} \mathrm{~W} / \mathrm{cm}^{2}\right)\end{array}$ & $\begin{array}{c}E_{\mathrm{a}} \\
\left(10^{-3} \text { a.u. }\right)\end{array}$ & $\begin{array}{c}E_{\text {0crit }}^{2}(\tilde{\omega}) / 4 \tilde{\omega}^{2} \\
\left(10^{-3} \mathrm{eV}\right)\end{array}$ \\
\hline $\mathrm{Na}_{8}$ & 2.64 & -0.470 & -0.031 & 0.501 & 1.41 & 0.66 & 17.5 & 1.44 \\
$\mathrm{Na}_{20}$ & 2.73 & 0.021 & 0.022 & 0.043 & 4.82 & 7.67 & 30.8 & 15.7 \\
$\mathrm{Na}_{40}$ & 2.72 & 0.022 & -0.100 & 0.078 & 3.58 & 4.23 & 37.0 & 8.64 \\
$\mathrm{Na}_{58}$ & 2.89 & 0.151 & -0.160 & 0.231 & 2.08 & 1.43 & 44.9 & 2.61 \\
$\mathrm{Na}_{92}$ & 2.98 & 0.128 & -1.589 & 1.461 & 0.83 & 0.23 & 50.1 & 0.39 \\
\hline
\end{tabular}

point, are presented in table 2 . It is instructive to compare the critical field $E_{0 \text { crit }}(\tilde{\omega})$ with the strength $E_{\mathrm{a}}$ of the internal anionic field. The latter can be related to the ionization potential $I_{\mathrm{a}}$ through $E_{\mathrm{a}}=\left(2 I_{\mathrm{a}}\right)^{3 / 2}$. It is seen that $E_{\mathrm{a}}$ exceeds $E_{0 \text { crit }}(\tilde{\omega})$ by the order of magnitude. The strong inequality $E_{\mathrm{a}} \gg E_{0 \text { crit }}(\tilde{\omega})$, being in accordance with the first condition from (5), justifies the approximation adopted in this paper to neglect the modification of the ground state orbitals due to the laser field. The last column in the table contains the values of ponderomotive energy of the photoelectron in the critical laser field. Comparing the ponderomotive shifts and the kinetic energies $\varepsilon=\tilde{\omega}-I_{\mathrm{a}} \sim 1$ $\mathrm{eV}$, one notices that the latter is larger by several orders of magnitude. Therefore, the second condition in (5) is also well fulfilled.

Hence, one can expect, that for the intensities $I_{0} \lesssim I_{0 \text { crit }}(\tilde{\omega}) \sim 10^{11} \mathrm{~W} / \mathrm{cm}^{2}$ the modification of the spectral-angular distribution due to the action of the field of the induced dipole moment can be quite pronounced. To carry out the corresponding quantitative estimates one can use the formulae obtained within the framework of perturbation theory.

The calculated profiles of the angular distributions are presented in figure 4. In the present paper we have not made an attempt to compute the angular asymmetry parameter $\beta(\tilde{\omega})$ for sodium anion clusters (neither have we found its values in the literature). Instead, for each target we carried out the calculations for the three values, $\beta=-1,0,2$, aiming to demonstrate that the modification of the profile takes place for any $\beta$ from the allowable interval $[-1,2]$.

In each graph from figure 4 the thick solid curve represents the unperturbed profile

$$
F_{\beta}(\theta) \equiv 1+\beta P_{2}(\cos \theta)= \begin{cases}\frac{3}{2} \sin ^{2} \theta & \text { for } \beta=-1 \\ 1 & \text { for } \beta=0 \\ 3 \cos ^{2} \theta & \text { for } \beta=2 .\end{cases}
$$

The emission angle $\theta$ is measured with respect to the vector $\mathbf{E}_{0}$, which is indicated in 


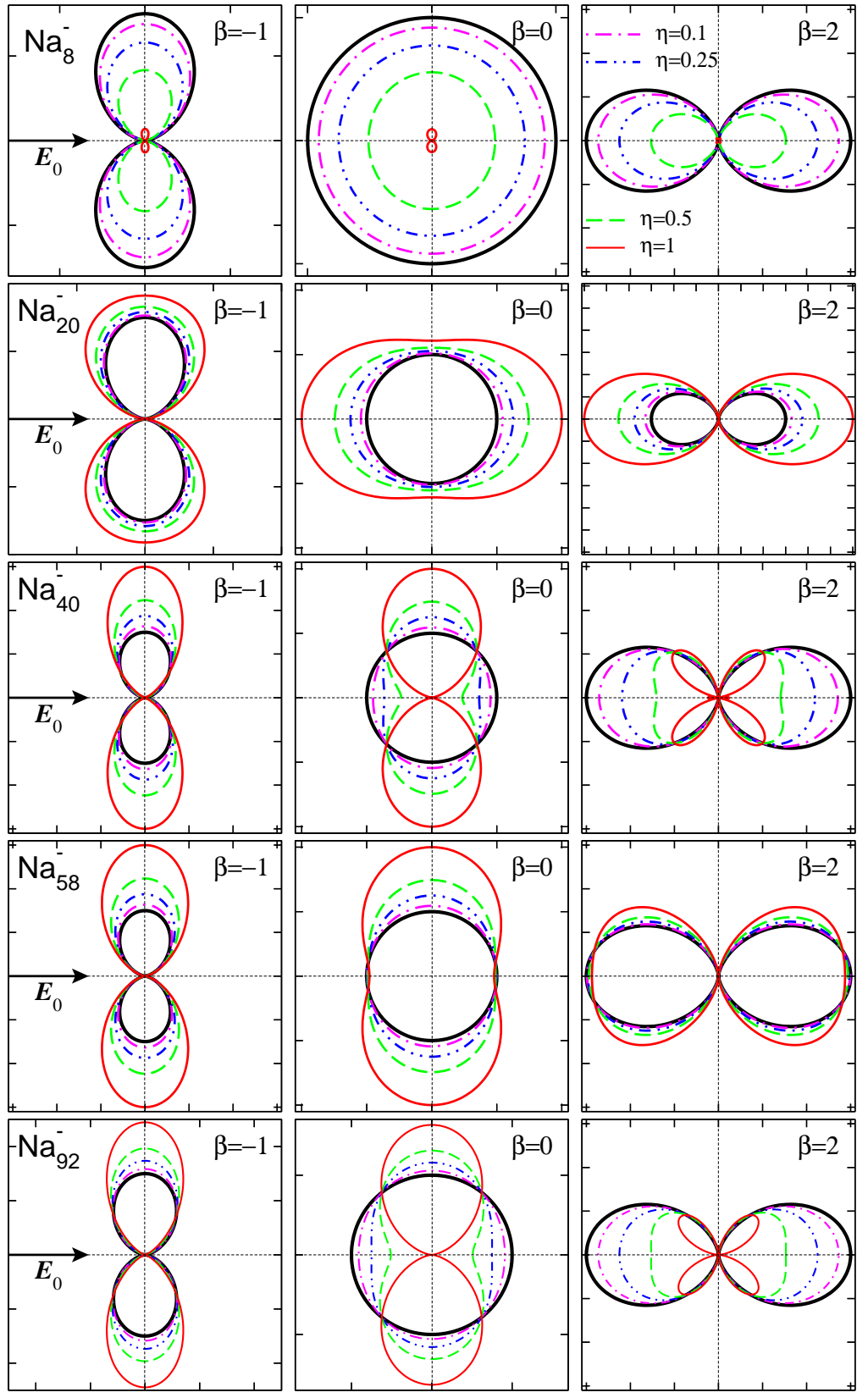

Figure 4. Profiles $\mathcal{F}_{\beta}(\theta)$ of the angular distribution (35) calculated for different sodium cluster anions and for the three values of asymmetry parameter $\beta$ (as indicated), and for several values of the ratio $\eta=I_{0} / I_{0 \text { crit }}$ (see the legend in the graph $\beta=2$ for $\mathrm{Na}_{8}^{-}$). The data refer to the photon energies $\tilde{\omega}$ indicated in table 2 . Thick black curves in each graph represent the field free distribution $F_{\beta}(\theta)$, eq. (34). The length of line segment connecting the origin and a given point on the curves is equal to the value of $\mathcal{F}_{\beta}(\theta)$ or $F_{\beta}(\theta)$ (in absolute units) in the corresponding direction. The emission angle $\theta$ is measured with respect to $\mathbf{E}_{0}$, which is shown in the graphs with $\beta=-1$. In each graph the spacing between ticks is equal to 1 for both directions.

the graphs with $\beta=-1$. Other curves correspond to the modified profiles

$$
\mathcal{F}_{\beta}(\theta) \equiv F_{\beta}(\theta)\left(1+E_{0}^{2}\left(\kappa_{0}+\kappa_{2} P_{2}(\cos \theta)\right)\right)=F_{\beta}(\theta)\left(1+\eta \frac{\kappa_{0}+\kappa_{2} P_{2}(\cos \theta)}{|\kappa|_{\max }}\right)
$$


(see (13), (27) and (33)) calculated for several values of the ratio $\eta=I_{0} / I_{0 \text { crit }} \leq 1$. For each anion the data refer to the photon energy $\tilde{\omega}$.

Figure 4 demonstrates, that even for comparatively low intensities (corresponding to $\eta \gtrsim 10^{-1}$ ) the changes in the profiles are already visible. For $\eta \leq 1$ they become much more pronounced. It is seen that variation of the profile depends, both qualitatively and quantitatively, on the value of $\beta$ and on the choice of the target anion which enters via the coefficients $\kappa_{0}$ and $\kappa_{2}$.

In the case $\beta=-1$ the field-free profile $F_{\beta}(\theta)$ is proportional to $\sin ^{2} \theta$ yielding $F_{-1}\left(0^{\circ}\right)=0$ as the minimum and $F_{-1}\left(90^{\circ}\right)=1.5$ as the maximum values within the interval $\theta=\left[0^{\circ}, 90^{\circ}\right]$. The modified profile can be written as $\mathcal{F}_{-1}(\theta) \propto \sin ^{2} \theta\left(a+b \cos ^{2} \theta\right)$ with $a=1+\eta\left(2 \kappa_{0}-\kappa_{2}\right) / 2|\kappa|_{\max }$ and $b=3 \eta \kappa_{2} / 2|\kappa|_{\max }$. One easily shows, that for $|a|<|b|$ the function $\mathcal{F}_{-1}(\theta)$ acquires additional extremum. However, this inequality cannot be met for the considered targets (see the values of $\kappa_{0}, \kappa_{2}$ and $|\kappa|_{\text {max }}$ in table 2 ) and for $\eta \leq 1$. Therefore, the pattern of the modified angular distribution is similar to that obtained without the influence of the induced dipole. Namely, the strongest emission occurs in the directions perpendicular to the field $\mathbf{E}_{0}$ and there is no emission for $\theta=0^{\circ}$ and $180^{\circ}$. On the whole, the distortion increases with $\eta$, attaining the largest values at $\theta=90^{\circ}$. The trend of the largest distortion depends on the sign of $\kappa_{0}$ : if $\kappa_{0}>0$ (as in the cases of all anions but $\mathrm{Na}_{8}$ ) then $\mathcal{F}_{-1}>F_{-1}$ whereas negative $\kappa_{0}$ leads to a shrinking of the modified profiles (as it happens for $\mathrm{Na}_{8}^{-}$).

For $\beta=0$ the unperturbed angular distribution is isotropic. The modified profile reads as $\mathcal{F}_{0}(\theta) \propto a+b \cos ^{2} \theta$, with $a$ and $b$ defined as above. The way the profile evolves with the increase of $\eta$ to a great extent depends on the sign of $\kappa_{2}$. For $\kappa_{2}>0$ the emission along $\pm \mathbf{E}_{0}$ dominates over the emission in the perpendicular directions (see the case of $\mathrm{Na}_{20}^{-}$), whereas for negative $\kappa_{2}$ the perpendicular direction becomes preferable. The graphs from the central column in figure 4 demonstrate, that for $\eta \approx 1$ the distortion can become dramatic both in form and in magnitude.

In the limit $\beta=2$ the modified profile behaves as $\mathcal{F}_{2}(\theta) \propto \cos ^{2} \theta\left(a+b \cos ^{2} \theta\right)$. The additional extrema appear if the following two conditions, $a b<0$ and $|a|<2|b|$, are met. These conditions can be satisfied in the limit $\eta \approx 1$ for all considered anions but $\mathrm{Na}_{20}^{-}$. Most explicitly it is seen in $\mathrm{Na}_{40}^{-}$and $\mathrm{Na}_{92}^{-}$graphs, where the curves with $\eta=1$ represent the four-petal angular distributions with the maximum emission at $\theta=45^{\circ}$ and $135^{\circ}$. The same pattern characterizes $\mathrm{Na}_{8}^{-}$, although in this case it is hardly visible due to a strong decrease in the magnitude of $\mathcal{F}_{2}(\theta)$ for $\eta=1$.

Finally, let us briefly discuss the influence of the induced dipole moment on the cross section integrated over the emission angles. Recalling (30) and (31), one finds the following formula for the ratio of the cross sections with, $\sigma(\omega)$, and without, $\sigma_{0}(\omega)$, account for the correction:

$$
\frac{\sigma(\omega)}{\sigma_{0}(\omega)}=1+E_{0}^{2}\left(\kappa_{0}(\omega)+\frac{\beta(\omega) \kappa_{2}(\omega)}{5}\right) .
$$

Figure 5 presents the ratios $\sigma(\omega) / \sigma_{0}(\omega)$ versus $\omega$. For each anion the ratio was calculated using the dependences $\kappa_{0}(\omega)$ and $\kappa_{0}(\omega)$, presented in figures 2 and 3 , and for three 

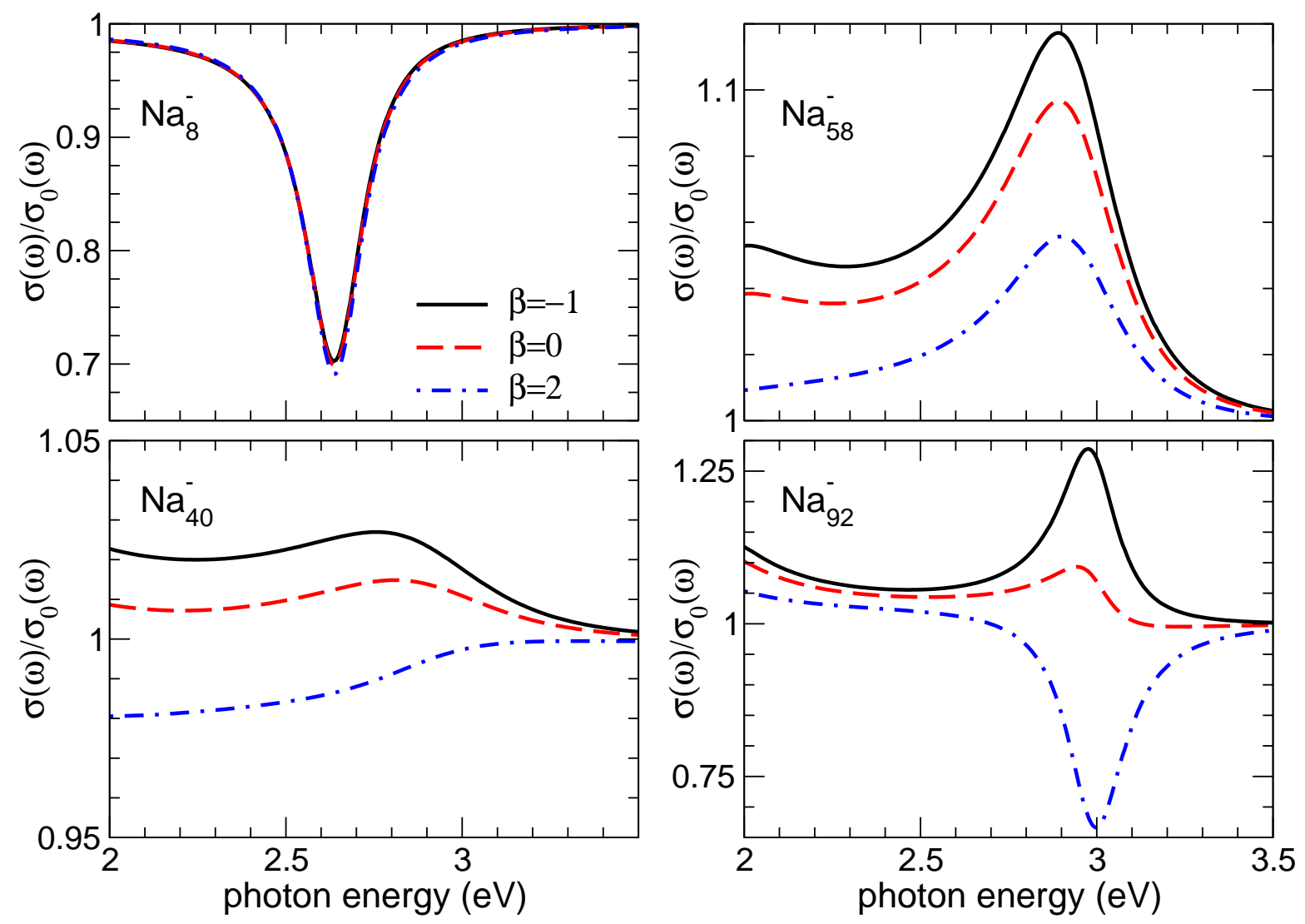

Figure 5. Ratio $\sigma(\omega) / \sigma_{0}(\omega)$ (see (36)) calculated for different sodium cluster anions and for the three values of asymmetry parameter $\beta$ (as indicated in the top left graph). The data refer to the laser field intensity $I_{0}=2.1 \times 10^{10} \mathrm{~W} / \mathrm{cm}^{2}$.

values of the asymmetry parameter (as indicated). The intensity of the laser field was set to $I_{0}=2.1 \times 10^{10} \mathrm{~W} / \mathrm{cm}^{2}$, which corresponds to the field strength $E_{0}=8 \times 10^{-4}$ a.u. Comparing the latter value to the critical field $E_{0 \text { crit }}(\omega)$ (see figures 2 and 3 ) one notices, that for all targets and in the whole range of $\omega$ the parameter $\eta=E_{0}^{2} / E_{0 \text { crit }}^{2}(\omega)$ is less than one. Thus, formula (36) is applicable for the quantitative estimate of the correction due to the induced moment.

As one can see from figure 5 , the largest deviation (up to $\approx 30 \%$ ) of $\sigma(\omega)$ from $\sigma_{0}(\omega)$ occurs for $\mathrm{Na}_{8}^{-}$and $\mathrm{Na}_{92}^{-}$in the vicinities of the plasmon resonances, where $\eta$ reaches its maximum values: $\eta \approx 0.57$ for $\mathrm{Na}_{8}^{-}$and $\eta \approx 0.95$ for $\mathrm{Na}_{92}^{-}$. In the case of $\mathrm{Na}_{92}^{-}$a strong inequality $\left|\kappa_{2}\right| \gg\left|\kappa_{0}\right|$ is valid (see figure 3 or/and table 2). Therefore, the magnitude of the correction term from the right-hand side of (36) becomes sensitive to the choice of the asymmetry parameter. As a result, the ratio $\sigma(\omega) / \sigma_{0}(\omega)$ strongly varies with $\beta$. On the contrary, for $\mathrm{Na}_{8}^{-}$the relation $\left|\kappa_{2}\right| \ll\left|\kappa_{0}\right|$ is valid, leading to a weak dependence of the ratio on $\beta$.

For the anions $\mathrm{Na}_{40}^{-}$and $\mathrm{Na}_{58}^{-}$the largest values of $\eta$ equal to 0.05 and 0.15 , correspondingly. Hence, the intensity $I_{0}$ is small compared to $I_{0 \text { crit }}(\omega) \propto E_{0 \text { crit }}^{2}(\omega)$. As a result, the deviation of $\sigma(\omega)$ from the field-free value $\sigma_{0}(\omega)$ is less pronounced. 


\section{Summary}

In summary, we have demonstrated that the dipole moment, induced at the residue by the incoming laser field, can strongly influence the photoionization process. The additional time-dependent long-range field due to the induced moment, acting on the escaping electron, modifies the spectral-angular and spectral distributions of photoelectrons.

The effect itself, as well as its quantitative treatment, is quite sensitive to the choice of the target and to the parameters of the laser field. We have demonstrated, that for a strongly polarizable target (e.g., metallic cluster anions) strong modifications of the characteristics of a single-photon ionization process in the vicinity of the plasmon resonance (typically, $\omega_{\mathrm{s}} \approx 2-3 \mathrm{eV}$ ) can be achieved by applying laser fields of comparatively low intensities $I_{0} \sim 10^{10}-10^{11} \mathrm{~W} / \mathrm{cm}^{2}$.

From a theoretical viewpoint, the advantage of this regime is that the field of induced dipole moment can be treated perturbatively, whereas the influence of the laser field on the photoelectron (a ponderomotive effect) and on the ground state of the target can be ignored. We have shown, that even in this weak-field regime one can expect dramatic changes in the profile of angular distribution of photoelectrons as well as in the $\omega$ dependence of the photoionization cross section. To a great extent, these changes depend on the parameters of the target, like its radius, the static and dynamic polarizabilities, the energy of the plasmon resonance peak and the affinity (or, the ionization potential in the case of a neutral target). Additional variety can be obtained by changing the intensity and the frequency of the laser field.

The analysis, which we have carried out, is based on simple models adopted for the description of the photodetachment process (the plasmon resonance approximation) and

of the interaction of the escaping electron with the static field of the residue (via the LDA + long-range polarization potential). We have also restricted ourselves to the case of spherically-symmetric targets. A more accurate quantitative treatment must include more sophisticated approaches, which take into account the many-body correlation effects intrinsic to both the photodetachment and the electron escape. However, these will leave untouched the physics behind the basic phenomenon discussed in our paper.

It is possible to study the predicted phenomena by means of modern experimental techniques. The latter include the production and manipulation of the beams of cluster anions of various types and sizes, the energy and angle resolved photoelectron spectroscopy, the availability of the laser fields of various intensity, frequency and pulse duration $[4,5]$. We believe, that with all these components at hand, soon it will become possible to test the theory against experimental data.

\section{Acknowledgments}

We are grateful to Roman Polozkov for the help in numerical calculation of the ground states of sodium clusters, and to Steven Manson and Bernd von Issendorff for the helpful 
discussions.

This work was supported by the European Commission within the Network of Excellence project EXCELL (project number 515703).

\section{References}

[1] P.A. Golovinski, I.Yu. Kiyan, 1990, Sov. Phys. - Uspekhi 33, 453.

[2] V.E. Chernov, I.Yu. Kiyan, H. Helm, B.A. Zon, Phys. Rev. A 71, 033410 (2005).

[3] G.F. Gribakin, M.Yu. Kuchiev, Phys. Rev. A 55, 3760 (1997).

[4] O. Kostko, C. Bartels, J. Schwöbel, C. Hock, B. v. Issendorff, J. Phys.: Conf. Series 88, 012034 (2007).

[5] O. Kostko, 2007, Photoelectron spectroscopy of mass-selected sodium, coinage metal and divalent metal cluster anions. Inaugural-Dissertation (Universität Freiburg).

[6] Gordon W. F. Drake (Ed.), 2006, Springer Handbook of Atomic, Molecular and Optical Physics (Germany: Springer).

[7] J. Cooper, R.N. Zare, 1968, J. Chem. Phys. 48, 942.

[8] D.A. Varshalovich, A.N. Moskalev, V.K. Khersonskii, 1988, Quantum Theory of Angular Momentum (World Scientific).

[9] J-P. Connerade, A.V. Solovyov, J. Phys. B: At., Mol., Opt. Phys 29, 365 (1996).

[10] L.G. Gerchikov, A.V. Solovyov, J-P. Connerade, Walter Greiner, J. Phys. B: At., Mol., Opt. Phys 30, 4133 (1997).

[11] L.G. Gerchikov, A.V. Solovyov, Z. Phys. D 42, 279 (1997).

[12] Atomic Clusters and Nanoparticles, NATO Advanced Study Institute, les Houches Session LXXIII, les Houches, 2000, edited by C. Guet, P. Hobza, F. Spiegelman and F. David (EDP Sciences and Springer Verlag, Berlin, 2001).

[13] C. Guet, W.R. Johnson, Phys. Rev. B 45, 11283 (1992).

[14] L.I. Kurkina, Phys. Solid State 43, 792 (2001).

[15] W.D. Knight, K. Clemenger, W.A. de Heer, W.A. Saunders, Phys. Rev. B 31, 445 (1985).

[16] T.R. Reiners, C. Ellert, M. Schmidt, H. Haberland, 1995, Phys. Rev. Lett. 74, 1558.

[17] C. Yannouleas, E. Vigezzi, R.A. Broglia, Phys. Rev. B 47, 9849 (1993).

[18] R.A. Molina, D. Weinmann, R.A. Jalabert, Phys. Rev. B 65, 155427 (2002).

[19] V.V. Babikov Sov. Phys. - Usp. 92, 271 (1967).

[20] F. Calogero, 1967, Variable Phase Approach to Potential Scattering (New York: Academic Press).

[21] O. Gunnarsson, B. I. Lundqvist, Phys. Rev. B 13, 4274 (1976).

[22] M. Bernath, O. Gragún, M.R. Spinella, H. Massmann, Z. Phys. D 33, 71 (1997).

[23] P. Descourt, M. Farine, C. Guet, J. Phys. B: At., Mol., Opt. Phys 32, 4565 (2000). 